\title{
Corrigendum to "Brain Activation and Psychomotor Speed in Middle-Aged Patients with Type 1 Diabetes: Relationships with Hyperglycemia and Brain Small Vessel Disease"
}

\author{
Misun Hwang, ${ }^{1}$ Dana L. Tudorascu, ${ }^{2}$ Karen Nunley, ${ }^{3}$ Helmet Karim, ${ }^{4}$ \\ Howard J. Aizenstein, ${ }^{5}$ Trevor J. Orchard, ${ }^{6}$ and Caterina Rosano ${ }^{7}$ \\ ${ }^{1}$ Department of Radiology, University of Pittsburgh, 3600 Forbes Avenue, Plaza Level, Pittsburgh, PA 15213, USA \\ ${ }^{2}$ Department of Internal Medicine, Department of Psychiatry, and Department of Biostatistics, University of Pittsburgh, \\ 200 Meyran Avenue, Suite 326, Pittsburgh, PA 15213, USA \\ ${ }^{3}$ Department of Epidemiology, University of Pittsburgh, 130 N. Bellefield Avenue, Suite 443, Pittsburgh, PA 15213, USA \\ ${ }^{4}$ Department of Bioengineering, University of Pittsburgh, 253 Sterling Plaza, Pittsburgh, PA 15213, USA \\ ${ }^{5}$ Department of Psychiatry, University of Pittsburgh, 3811 O'Hara Street, Pittsburgh, PA 15213, USA \\ ${ }^{6}$ Department of Epidemiology, University of Pittsburgh, 3512 Fifth Avenue, Pittsburgh, PA 15213, USA \\ ${ }^{7}$ Department of Epidemiology, University of Pittsburgh, 130 N. Bellefield Avenue, Suite 467, Pittsburgh, PA 15213, USA
}

Correspondence should be addressed to Caterina Rosano; car2350@pitt.edu

Received 17 October 2016; Accepted 25 October 2016; Published 16 March 2017

Copyright (C) 2017 Misun Hwang et al. This is an open access article distributed under the Creative Commons Attribution License, which permits unrestricted use, distribution, and reproduction in any medium, provided the original work is properly cited.

In the article titled "Brain Activation and Psychomotor Speed in Middle-Aged Patients with Type 1 Diabetes: Relationships with Hyperglycemia and Brain Small Vessel Disease" [1], there was an error in the subheadings of Table 3. The phrase "response time" should be replaced with the phrase "main effect." The correct table is shown below. 
TABLE 3: Regions with functional activation that were correlated with performing the task in the scanner (main effect): positive associations are listed first and negative associations are listed next.

\begin{tabular}{lccc}
\hline Regions & Cluster size & $\begin{array}{c}\text { Peak } T \text {-Score } \\
(\mathrm{df}=84)\end{array}$ & $\begin{array}{c}\text { Montreal Neurological Institute } \\
\text { coordinate for Peak } T \text {-Score }\end{array}$ \\
\hline Superior parietal lobe, bilaterally & Regions with activation positively correlated with main effect & $-28,-62,52^{1}$ \\
Left middle frontal gyrus & 6912 & 12 & $-44,2,34^{2}$ \\
Right middle frontal gyrus & 3529 & 12 & $36,2,52^{3}$ \\
Medial frontal gyrus, bilaterally & 3133 & 8.5 & $2,20,46^{4}$ \\
Right inferior frontal gyrus & 1196 & 8.6 & $34,24,-5^{5}$ \\
Right thalamus & 375 & 6.3 & $18,-6,19$ \\
Left thalamus & 214 & 4 & $-16,-12,16$ \\
& 212 & 4.1 & $-66,-22,1$ \\
Left superior temporal gyrus & Regions with activation negatively correlated with main effect & -6.6 & $-5,-30,22$ \\
Right superior temporal gyrus & 1285 & -5 & $-4,-52,28$ \\
Posterior cingulate cortex, bilaterally & 891 & -6.1 & $-2,64,1$ \\
Left medial frontal gyrus & 821 & -5.1 & $48,-26,64$ \\
Right postcentral gyrus & 420 & -5.9 & \\
\hline
\end{tabular}

This table reports the spatial distribution of the mean group activation (obtained from the DSST > control condition contrast and from the control condition $>$ DSST contrast), including the size of cluster, the maximum $Z$ statistic for the cluster, and the location of the maximum $Z$ statistic in Montreal Neurological Institute coordinates. The corrected alpha is the probability of false positive detection based on the combination of individual voxel probability thresholding and minimum cluster size thresholding.

${ }^{1}$ This cluster extends medially to include the precuneus and caudally to include the superior occipital gyrus; it includes the most dorsal part of the inferior parietal lobule.

${ }^{2}$ This cluster extends rostrally to include the supplementary motor area, caudally to include the precentral gyrus, and medially to include the insula.

${ }^{3}$ This cluster extends rostrally to include the supplementary motor area, caudally to include the precentral gyrus, and ventrally to include the inferior frontal gyrus.

${ }^{4}$ This cluster extends caudally in the right hemisphere to include the dorsal cingulate cortex.

${ }^{5}$ This cluster covers part of the insula.

\section{References}

[1] M. Hwang, D. L. Tudorascu, K. Nunley et al., "Brain activation and psychomotor speed in middle-aged patients with type 1 diabetes: relationships with hyperglycemia and brain small vessel disease," Journal of Diabetes Research, vol. 2016, Article ID 9571464, 11 pages, 2016. 


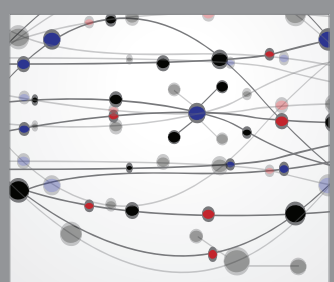

The Scientific World Journal
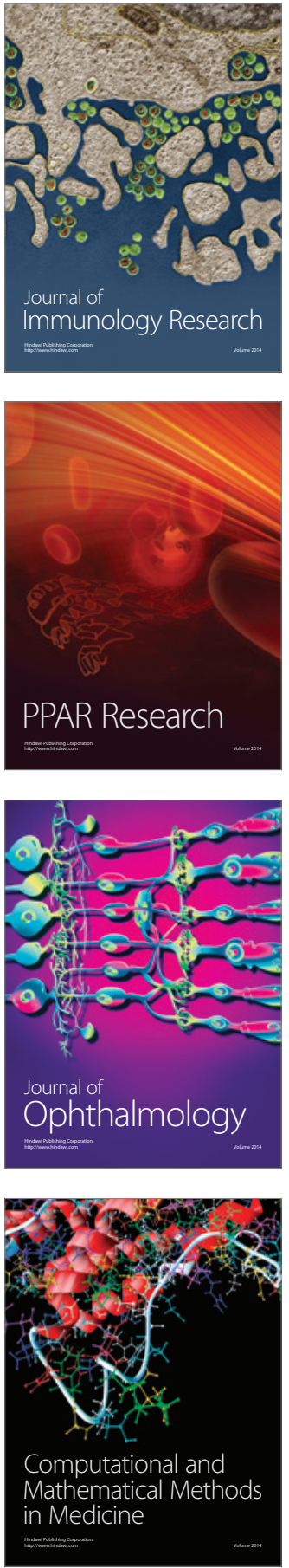

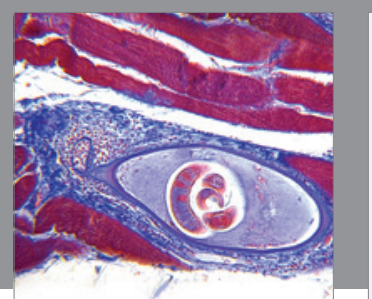

Gastroenterology Research and Practice
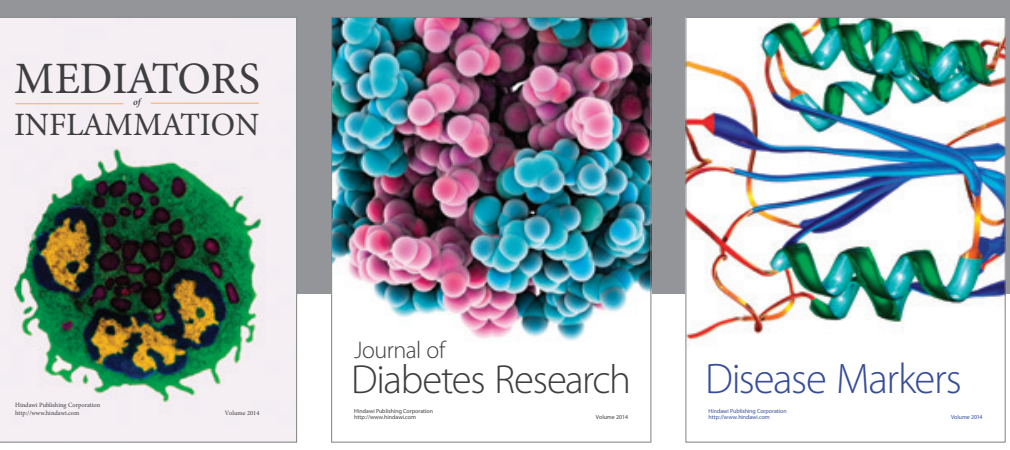

Disease Markers

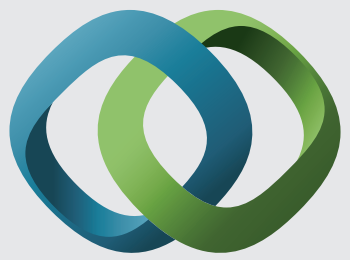

\section{Hindawi}

Submit your manuscripts at

https://www.hindawi.com
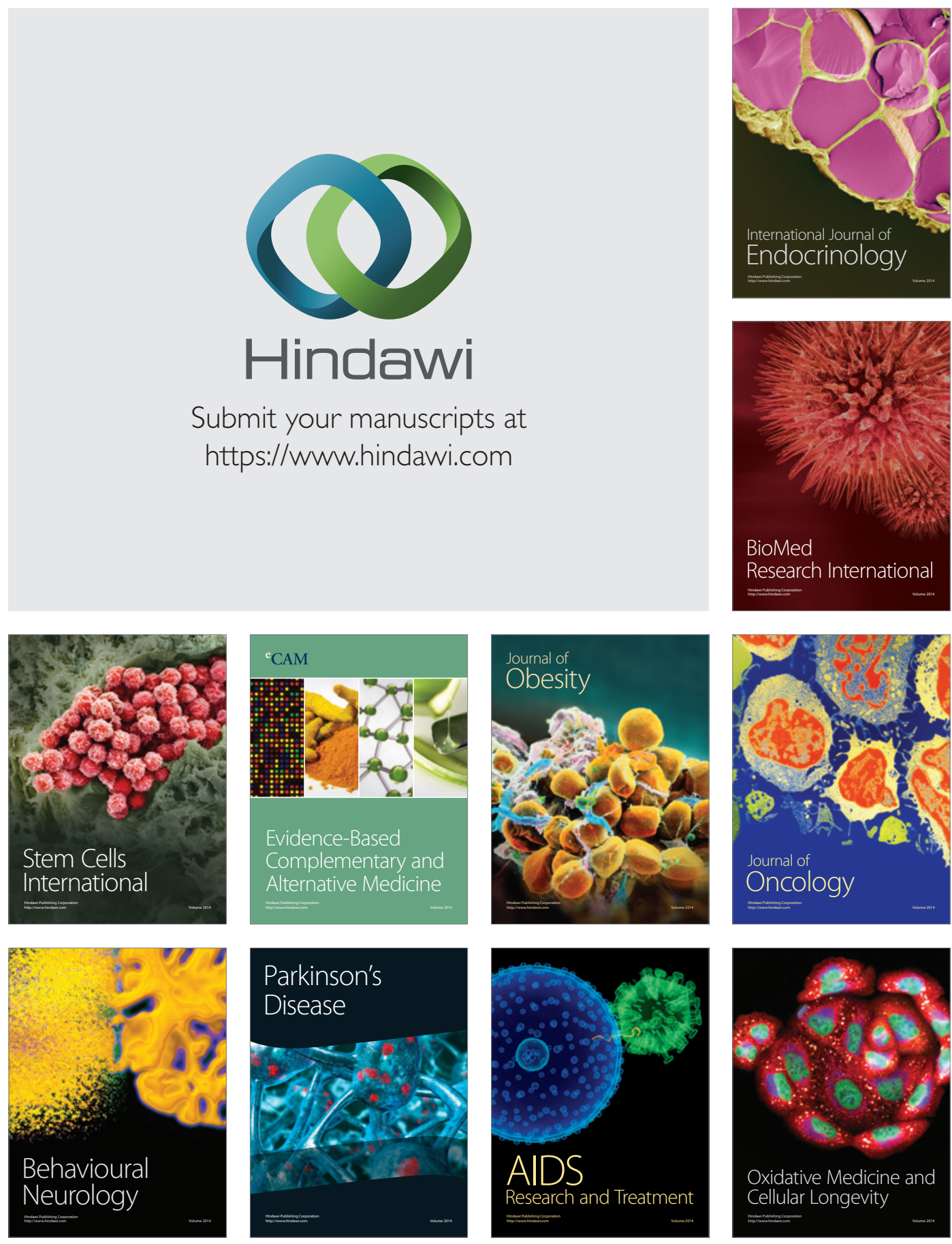